\title{
Man in the Middle of Face Recognition System: using Skin Color and Template
}

\author{
T. Archana, T. Venugopal
}

\begin{abstract}
During last 10 years people are very much attracted to face recognition systems and they are very much eager to solve the issues related to face recognition system. It helped them very much in the field of electronics and uses over pattern unlocking and password entering system. There are numerous applications as for security, affectability and mystery. Detection of a face is the most significant and initial step of recognition framework. This article demonstrates a new method to face recognition system using color and template of an image. Whatever the background it may go to be, our system will detect the face, which is an important stage for face detection. The pictures utilized in this framework for Face detection are the color images, while the images used for the Face Recognition are the Gray images which are converted from color pictures.

The illumination compensation technique is applied on all the images for removing the effect of light. The Red, Green, and Blue values of each pixel will be converted to $\mathrm{YCbCr}$ space. Based on the probability of each pixel in terms of $\mathrm{Cb}, \mathrm{Cr}$ values, we extract the skin pixels from the query image. The positive probability shows a "skin pixel", while the negative probability shows "not a skin pixel". Finally the face is projected. In face recognition, we used 4 templates of different sizes for Gabor image content extraction. Finally we employed the relevance feedback mechanism to retrieve the most similar images. If the user did not satisfy with the given results he can give the correct images to the system from the displayed images. Exploratory outcomes demonstrate that the demonstrated system is adequate to recognize face of a human face in a picture with an exactness of $94 \%$.
\end{abstract}

Keywords - Probability function, Face detection, Gabor based templates, templates extraction, Face Recognition

\section{INTRODUCTION}

There are numerous computations are involved in the face detection. Just to identify the face in the image, there needs so many calculations to be occurred relating to the tone values of that image. Color is a huge part of people's countenances. Even though usage of skin color in dealing with the face, contains so many problems because different people from different areas will have different colors of faces. But accepting color as the key part for identification of face will reach us to the destination very easily. In some situations color acts as the direction invariant. This key feature of the

Revised Manuscript Received on December 30, 2019.

* Correspondence Author

T. Archana*, Assistant Professor, Department of CSE, University College of Engineering KU, Kothagudem (Telangana) India.

E-mail: archanapraneeth@gmail.com

Dr. T. Venugopal, Professor, Department of CSE, JNTUH College of Engineering Jagityal Nachupally, Karimnagar (Telangana) India.

E-mail: t_vgopal@rediffmail.com

(C) The Authors. Published by Blue Eyes Intelligence Engineering and Sciences Publication (BEIESP). This is an open access article under the CC BY-NC-ND license (http://creativecommons.org/licenses/by-nc-nd/4.0/) color helps us to solve the color related problems easily. Regardless, color is not a physical thing to touch and catch. It is a wonder as we feel it is a supernatural characteristic of electromagnetic radiation with different wavelengths in colors striking the retina. There is couple of issues with the light, like the photo that was taken by a camera will be different and a photo taken by a mobile will be different. So it is important to deal with the color related issues, as we know color changes with the effect of light. So if we want to use color as a component, we have to come across various issues. Changes on the face will change the color on the face, one weakness of the color is, it is easily affected with the brightness changes.

Face identification has gotten huge enthusiasm from PC researchers and therapists over late decades, to a great extent operated by its potential in all the whereas where it is used, for example, people PC cooperation, video observation, and mixed media content investigation. Albeit numerous frameworks for FER [1] have been proposed and executed, by far most of the people utilize non blocked facial pictures which are clicked under some specific i.e. controlled research facility conditions [1]. Building powerful Face Recognition frameworks under changes on the face is as yet a difficult undertaking. Facial impediments can be two types: brief and precise [2]. Brief impediment can result from a piece of the face clouded by a hand or an item or attributable to head development. Precise impediment comes about because of wearing a thing, for example, shades, a scarf or cover. Not at all like different corruptions, for example, present varieties, whose qualities can be surmised previously, facial impediment is especially hard to deal with because of its "arbitrary" trademark: impediment can happen indiscriminately positions and blocking patches can be subjectively enormous in size. State of the art FR examines on taking care of impediment attempt to remake the blocked geometric or surface highlights and visual properties of people's face. Techniques involving remaking geometry incorporate head segment investigation (PCA) [2], the developed Kanade Lucas tracker [3], [4], Bayesian tracker [5], and the transferable conviction model [6], and those for reproducing surface lean on vigorous PCA [7], [8]. These frameworks depend vigorously on precise recognition of geometric focuses and impediment locales. All of them do not have the ability to completely catch the provincial surface highlights or lose a piece of neighborhood surface data because of the recreation mistake. Nearby surface data is significant for FER. Examinations concerning the impact of impediment on acknowledgment execution have may be utilized a blend of structure of shape and surface highlights [9], [10], or drawn closer Face Expression Recognition from the perspective on people judgement [11], [12]. 
As these things have given helpful knowledge, for example, the most discriminative facial territories for explicit articulations, they have been confined to impediment over determined districts, for example, the mouth, eyes, and nose. To display the "irregular" normal for impediment in reality, it is alluring to test with impediment at arbitrary areas and blocking patches of various sizes. In addition to that, most preparing and testing informational collections utilized in these examinations were coordinated and have been both blocked in a similar way. It is important to test the acknowledgment execution on blocked pictures when non-impeded pictures are utilized for preparing (crisscrossed conditions). This is especially significant for certifiable applications in that it can dispense with the progression of producing the blocked preparing pictures by mentioning customers at the hour of enrolment. It isn't essentially conceivable to acquire preparing pictures with every conceivable kind of impediment. In this work, another methodology is received and there is no endeavor to reproduce blocked pieces of a face. Rather, a strong Face Expression Recognition approach utilizing arbitrarily inspected Gabor-based templates is demonstrated. These templates are removed by a calculation and fill in as a pool of neighborhood highlights; along these lines, just a piece of them are affected by impediment. Template matching is utilized to locate the most comparative highlights situated inside a space around these templates.

This article is structured as, related work is discussed in section 2, proposed system using skin color and template based face recognition is discussed in section 3 , experiment setup and results are discussed in section 4. Finally, conclusion is given in $5^{\text {th }}$ section.

\section{RELATED WORK}

In this related work, we will discuss about Color models for Face Detection and various methods for Face Recognition.

\section{A. Color Models for Face Detection}

In this section, we will discuss about the red Green Blue color model and Red Green Blue Model for Face Detection and YCbCr color space and also Algorithm for Face Recognition in Space

\section{a. Red Green Blue Color Model}

RGB relies upon the coordinate structure which is a guide solid shape as showed up in Fig.1. The square has the Red Green Blue regards in the three corners - colors like magneta, cyan, yellow and the other three corners the exceptionally differentiating at the root is at the corner most remote from the beginning. The dark scale is arranged in stake joining high complexity. They are called added substance "fundamental" considering the way that the colors are added to convey the perfect color. As a result of the high connection between's color portions: blue, green, and red as each fragment is obligated with the influence of luminance of the light power of nature, so gets frustration concerning many picture getting ready applications. In utilitarian, this model isn't fitting to portray colors similarly as human comprehension [13].

\section{b. Red Green Blue Model for Face Detection}

Retrieval Number: B4028129219/2019@BEIESP

DOI: 10.35940/ijeat.B4028.129219

Journal Website: www.ijeat.org
Skin tone is the significant component of the face and is one of a kind as a result of its color fixings. Skin tone pixels will be effectively recognized utilizing standardized color histogram which is standardized for each future change in the power of luminance of the division. Furthermore, consequently RGB vector is changed into a vector $[\mathrm{r}, \mathrm{g}]$ color standard which thusly gives quick methods for distinguishing the skin. This gives the skin color area, which limits face as in eqn 1 and 2 . As shown early RGB experiences the impact of luminance, however is as yet ready to enable us to take out certain colors which are unmistakably outside the extent of typical skin color. After the investigation of various degrees of the Red Green Blue color space, one thing was discovered that the accompanying principle functions admirably in disposing of some excess pixels that are named as non-face [14].

$$
\begin{gathered}
0.836 G-14<B<0.836 G+44=>\text { Skin } \\
0.79 g-67<B<0.78 g+42=>\text { Skin }
\end{gathered}
$$

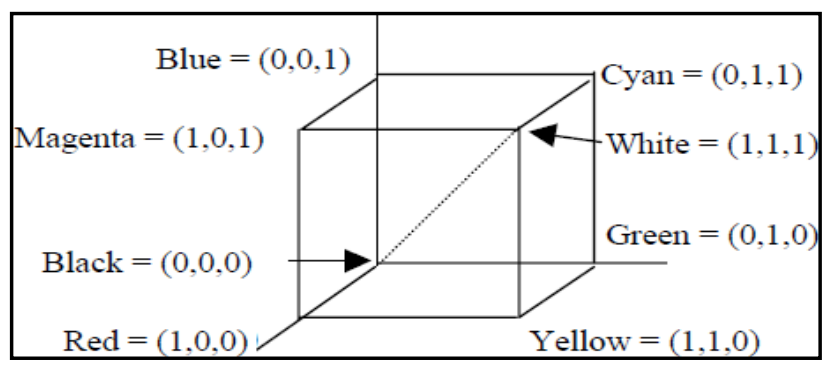

Figure 1 Red Green Blue color values on the cube

\section{c. Color Space: YCbCr}

YCbCr color space was portrayed to fulfill the creating need of cutting edge planning figurings of video information and has ended up being extensively used color space in modernized chronicles. It contains three sections, one section is the chrominance and other section is the luminance. TV transmission color space uses this model close by YUC and YIQ and is expected for space basic PAL and NTSC [14].

YCbCr color model was found out to allow the transmission of color data on TVs recalling that the present TV in profoundly differentiating still shows pictures in shades of dim, has the typical for luminance and separate color data, and it is utilized in various areas, for instance, pressure.

\section{d. Algorithm: Face Recognition in $\mathrm{YCbCr}$ Space}

In this model, the skin color pixels having a spot with the district show relative $\mathrm{Cb}$ and $\mathrm{Cr}$ regards as it is the skin color model reliant on the $\mathrm{Cr}$ and $\mathrm{Cb}$ values which can give incredible consideration of human races. YCbCr signals that are produced using the relating gamma adjusted RGB source using two described constants $\mathrm{KB}$ and $\mathrm{KR}$. After gamma review and before scaling and parities $\mathrm{YCbCr}$ sign are called Y', PB, and PR and are portrayed as showed underneath in eqn 3,4 , and 5 .

$$
\begin{gathered}
Y^{\prime}=K R * R^{\prime}+(1-K R-K B) * G^{\prime}+K B * B^{\prime} \\
P B=1 / 2 *\left(\left(B^{\prime}-Y^{\prime}\right) /(1-K B)\right) \\
P R=1 / 2 *\left(\left(R^{\prime}-\right.\right. \\
\left.\left.Y^{\prime}\right) /(1-K R)\right)(5) \\
\begin{array}{l}
\text { Published By: } \\
\text { Blue Eyes Intelligence Engineering } \\
\text { \& Sciences Publication }
\end{array}
\end{gathered}
$$


Here, gamma rectification is being utilized, therefore $\mathrm{R}^{\prime}, \mathrm{G}^{\prime}$ and $\mathrm{B}$ ' ostensibly territory from 0 to 1 ,

with 0 speaking to the base force (e.g., for show of the color dark) and 1 the most extreme (e.g., for show of the color white). From this the subsequent luminance signal (Y) worth will at that point have an ostensible territory from 0 to 1 . The chroma esteem (PB and $\mathrm{PB}$ ) qualities will at that point have an ostensible territory ranging -0.5 to +0.5 .

In dealing with the signals in modern structure, modifying off and scaling and balances are incorporated. For instance scaling and counterbalance associated with the $\mathrm{Y}^{\prime}$ part per assurance brings about the estimation of 16 for dark and the estimation of 235 for white when using a 8-piece portrayal. The standard has 8-piece digitized adjustments of CB and CR scaled to a substitute extent of 16 to 240. Thusly, rescaling by the division (235-16)/(240-16) equals to 219/224 is required in color system or taking care of in $\mathrm{YCbCr}$ space, driving quantization twists if the dynamic planning isn't occur using higher piece profundities.

The $\mathrm{Y}^{\prime} \mathrm{CbCr}$ that was characterized for standard-definition TV use in the ITU-R BT.601 standard for use with computerized part video is gotten from the comparing RGB space as seeks after: KB equals to 0.114 , KR equals to 0.299 . From the above constants and conditions, the going with can be surmised for ITU-R BT.601. Straightforward YPbPr rom basic $\mathrm{R}^{\prime} \mathrm{G}^{\prime} \mathrm{B}$ ' is resolved as seeks after in eqn. (6), eqn. (7), and eqn. (8).

$$
\begin{aligned}
& Y^{\prime}=0.299 * R^{\prime}+0.587 * G^{\prime}+0.114 * B^{\prime} \\
& P B=-0.168739 * R^{\prime}-0.331264 * G^{\prime}+0.5 * B^{\prime} \\
& P R=0.5 * R^{\prime}-0.418688 * G^{\prime}-0.81312 * B^{\prime}
\end{aligned}
$$

Computerized $\mathrm{Y}^{\prime} \mathrm{CbCr}$ is derived from analogue $R^{\prime} G^{\prime} B^{\prime}$ as pursues in eqn. (9), eqn. (10) and eqn. (11).

$$
\begin{array}{r}
Y^{\prime}=16+\left(\begin{array}{r}
65.481 * R^{\prime}+128.553 * G^{\prime}+24.966 * \\
\left.B^{\prime}\right)
\end{array}\right. \\
P B=128+\left(-37.797 * R^{\prime}-74.203 * G^{\prime}+\right. \\
\left.112.0 * B^{\prime}\right) \\
P R=128+\left(\begin{array}{c}
112.0 * R^{\prime}-93.786 * G^{\prime}+18.214 * \\
\left.B^{\prime}\right)
\end{array}\right.
\end{array}
$$

Please keep in mind that, resultant sign will be lying in between 16 to 235; the qualities in the range 0 to 15 they are called as foot room, and the qualities ranging from 236 to 255 are called head room.

Then again, advanced $\mathrm{Y}^{\prime} \mathrm{CbCr}$ is gotten from computerized Rd Gd Bd (8 bits for each example) as indicated by the accompanying conditions: (as in eqn. (12), eqn. (13), eqn. (14), eqn. (15), eqn. (16), and eqn. (17).

$$
Y^{\prime}=16+(65.481 * R d+128.553 * G d+
$$

$$
24.966 * B d) / 256
$$

$$
\begin{aligned}
& P B=128+(-37.797 * R d-74.203 * G d+ \\
& \left.112.0 * B^{\prime} d\right) / 256
\end{aligned}
$$

$$
\begin{gathered}
P R=128+(112.0 * R d-93.786 * G d-18.214 * \\
B d) / 256 \\
Y^{\prime}=16+(0.255 * R d+0.5021 * G d+0.0975 * \\
B d)
\end{gathered}
$$

$$
\begin{array}{r}
P B=128+(-0.148 * R d-0.368 * G d+0.439 * \\
\left.B^{\prime} d\right)
\end{array}
$$

The above conditions are used in the programming, some part of the proposed count. $\mathrm{Cr}$ and $\mathrm{Cb}$ are used instead of $\mathrm{PB}$ and PR. Resulting to inspecting and attempting various things with various breaking points, lastly it was found that best results were found out by using the going with declaration and by and large acknowledge that it isn't skin and may be ousted from additionally thought [15].

Lastly, we can order the pixel as skin pixel, if the Cb esteem lies in the range given in Eq. (18).

$$
102<C b<128=>\text { Skin }
$$

As of now we have seen the related work for Face Detection. Now we will discuss the methods developed for the Face Recognition through the history.

\section{B. Various Methods for Face Recognition}

\section{a. Template based Methods}

Template matching is theoretically identified with comprehensive methodology which endeavors to distinguish faces utilizing worldwide portrayals (J. Huang, in 1998). These kinds of techniques approach the face picture all in all and attempt to concentrate highlights from the entire face locale and afterward characterize the picture by applying an example classifier. One of the strategies used to concentrate includes in a holistic system, depends on factual methodologies which are examined in the accompanying area.

\section{b. Approaches: Statistical based}

A few methods that distinguish, parameterize and break down straight subspaces. Other than direct subspaces there are some measurable face recognition methods which depend on non-straight subspaces (like part PCA and piece LDA), change (like DCT, DCT and HMM and Fourier Transform) and Support Vector Machine (SVM).

\section{c. Approaches: Neural Network based}

Neural Network (in 1999 B. Yegnanarayana in ref [16]) is a best apparatus for design acknowledgment issues. In Kohonen's acquainted guide (in 1998, T. Kohonen, in ref [17]), perhaps the most punctual showing of neural system for face picture review applications is accounted for. Utilizing a little arrangement of face pictures, exact review was accounted for in any event, when information picture is extremely uproarious, low goals and measurement or when bits of the pictures are absent. A couple Neural Network based face recognition systems are talked about in the accompanying.

\section{d. Approach: Neural Network using single Adaptive layer}

A solitary layer versatile Neural Network (one for every individual) for face recognition, demeanor examination and face confirmation was accounted for in (T. J. Stonham, in 1984 as shown in ref [18]). A framework named Wilke, Stonham's recognition devise (WISARD) was conceived. 
It needs commonly 200-400 introductions for preparing every classifier where the preparation examples included interpretation and distinguishing proof in outward appearances.

\section{e. Approach: Multilayer Perceptron}

A large portion of the present literary works on face acknowledgment framework with neural systems present outcomes with few classes (frequently underneath 20). In (in 1993, D. Demers, in ref [19]) the initial 50 head parts of the face pictures were separated and decreased to 5 values utilizing auto cooperative neural network architecture. The subsequent portrayal was grouped utilizing a multilayer perceptron approach.

\section{f. Approach: Hop-field memory model:}

In (in 1998, Y. Dai, in ref [20]), a Hop-field memory model for the facial pictures is sorted out and the ideal methodology of learning is resolved. A strategy for face acknowledgment utilizing Hop-field memory model joined with the example coordinating is proposed. It shows better execution of database having 20 countenances of 40 subjects.

\section{PROPOSED SYSTEM}

This is our proposed system that gave us best results for face recognition. Our proposed system detects the face of a person by color based approach and it will recognize the face of a person by template based approach. It will recognize the images even with some changes and expressions in the face also. The stages in our proposed system are: firstly, it will capture the image. Secondly, the captured image will be provided with illumination compensation. Thirdly, the system will detect the face from the image through color based approach. Fourthly, that image will be sent for formation of Gabor filter images. And Fifthly, Templates will be extracted from Gabor filter images. These all the above said five phases will happen for all the images in the database for storing their template's values. When the user wants to receive the similar images from the database, then the matching of the query image template with the database images templates will take place. The architecture of the proposed system is shown in Fig.2. Fig.2 clearly explains the various stages in our proposed system.

Proposed system algorithm is discussed below, which is divided into four stages.

\section{Preprocessing Stage}

Step1: Input query image will be given by the user to the demonstrated system,

Step2: Calculate the probability of each pixel of query image using Eq. (26). The procedure is discussed in the Algorithm 1. Step3: Identify all the pixels with positive probability value. Pixels with positive probability value show the skin pixels.

Step4: Place these pixels in different groups (Group1, Group $2, \ldots)$. All the adjacent pixels lie in one group. Make all adjacent pixels as one group and other adjacent pixels as another group.

Step5: Draw rectangles around these groups. Find out the area of all the rectangles. Extract the rectangle with maximum area. This area will be the face area. See Figure 6 .
Step6: Develop/implement the Gabor filters by using Eq. (26) and Eq. (27) in the section c (Gabor filters).

Apply Gabor filters on the given query image, after applying it we will get set of Gabor images.

Step7: Extract the templates from the set of Gabor images obtained from the above step6, using the procedure discussed in the section d (Extraction of Template) and observe the Figure. 6.

Step8: Calculate the average of all templates (for all Gabor scales, orientations and templates for the both input and Database images) as shown in the Figure. 6.

\section{Similarity Measure Stage}

Step9: Calculate the distance between the query and DB images using Euclidean distance as given in the Eq. (1).

$$
d(I, Q)=\sqrt{\sum_{i=1}^{n}\left(I_{i}-Q_{i}\right)^{2}}
$$

Where $I_{i}$ and $Q_{i}$ are Average values of $I$ (DB image) and of $Q$ (Query image) respectively.

\section{Display Result Image Set.}

Step10: Most relevant Images will be displayed to the client based on the distance measure from the above step6. Which are having less distance will be displayed first, next less distance images will be displayed afterwards.

Step11: The quantity of pictures showed will rely upon the size of the showcase window.

\section{Feedback Process Stage}

Step12: Check client satisfaction, if client satisfies with result, stop the procedure. Otherwise, ask him for the feedback.

Step13: Now the client will tick mark the related images from the retrieved images (images obtained in step 7). And leave the irrelevant images.

Step14: Now the system takes the average values of relevant images and stores them in the Average values buffer (AVB).

Step 15: And now for the next iteration the system takes the query image given by the user. This query image may be same or different depending on the user.

Step16: Now the query average is compared with the Average values buffer (AVB). If the distance between them is zero, then only those images will be displayed in Step18. 


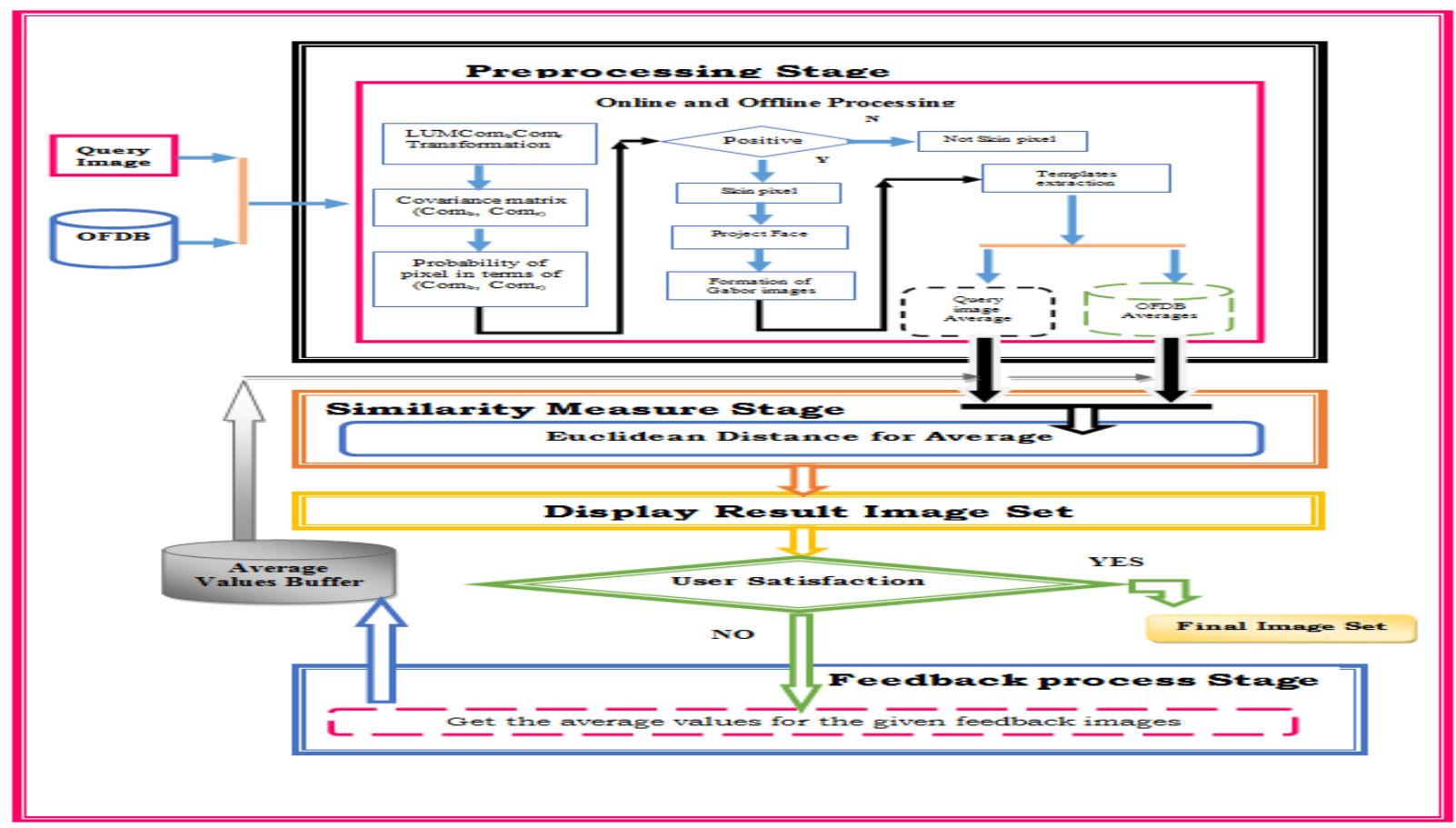

Figure 2 Detailed Proposed System Architecture

Step 17: Now the query average is compared with the average values present in the Only Face Database (OFDB). The less distance image will be displayed first and the next less distance images will be displayed afterwards.

Step 18: Now the system will display all the related images obtained in step16 and step17. Step16 images will be displayed first and Step17 images will be displayed next.

Step 19: Now the System asks client whether he is satisfied or not. If he is satisfied with the result set, then stop the process. Otherwise, The System asks him to tick mark the related images.

Step20: Now the system will collect the average values of the tick marked images and stores them in the Average Values Buffer (AVB).

Step21: And now for the next iteration the system takes the query image given by the user. This query image may be same or different depending on the user.

Step22: Now the query average is compared with the Average values buffer (AVB). If the distance between them is zero, then only those images will be displayed.

Step23: Now the System compares the query average with the average values present in the Only Face Database

(OFDB). The less distance image will be displayed first and the next less distance images will be displayed afterwards in Step24.

Step24: Now the system will display all the related images obtained in step 17 and step 18.

Step25: Now go to Step12 and continue from there.

\section{A. Preprocessing Stage}

In this stage, the query image will go under various phases to find out the face located in the picture. At first the client will give the input image to the system. After the user gives the query image to the system, it will firstly resize the image to $226 * 226$. Then it will be sent for the illumination compensation. The illumination compensation removes the light effect from the image. Then after that, the image will be sent for transforming it into $\boldsymbol{Y C} \boldsymbol{b} \boldsymbol{C r}$ color model. Now, the system will take the $\mathrm{Cb}$ and $\mathrm{Cr}$ values of each pixel and it will find out the mean of $\mathrm{Cb}$, and $\mathrm{Cr}$ values in the total image. Then it will find out the covariance matrix for the $\mathrm{Cb}, \mathrm{Cr}$ values. The found out values will be used in the probability function to identify the skin pixel in the query image. All the identified skin pixels will be projected as a face, and for this face we apply Gabor filters to obtain the Gabor images. Templates will be extracted from these Gabor images and then average for query image will be calculated.

\section{a. Color based approach for Face Detection}

The Face detection system stages are clearly given below in the Figure 3. Even though there are many methods to detect the face, we are using only color model because it only extracts the skin pixels from the image, whereas when we notice the other methods, they will extract the total face which will contain hair, and some portion of the shirt.

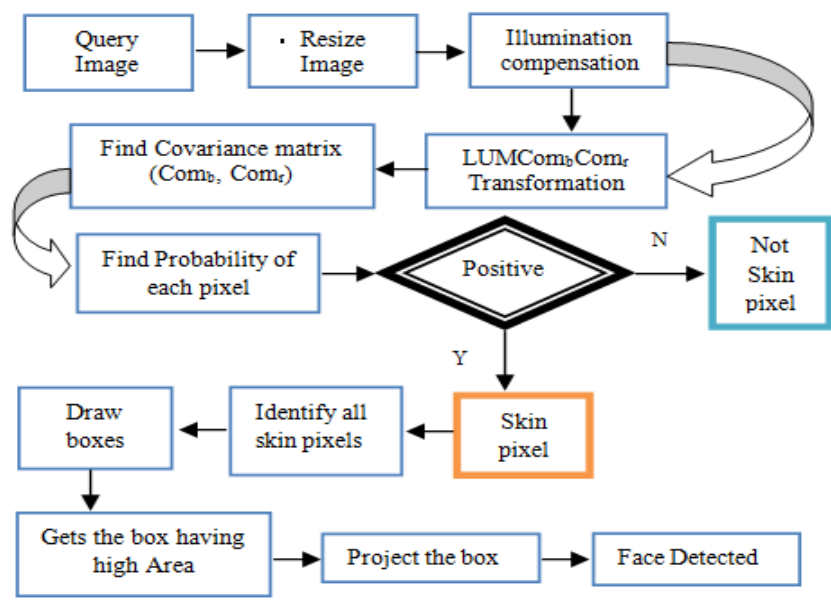

Figure 3 Proposed method for Face detection

\section{Algorithm 1. Proposed Face Detection}

Input: Color Image

Output: Detected Face Begin

Published By: 
Step1: In $\mathrm{LUMCom}_{b} \mathrm{Com}_{r}$ colorspace, for every pixel, there will be $\mathrm{Com}_{b}$ and $\mathrm{Com}_{r}$ values present. These values are very much helpful to find out the skin region. So after the conversion of $R, G, B$ color model to $\mathrm{LUMCOm}_{b} \mathrm{Com}_{r}$ color model, we will operate on skin color pixels in the form of $\mathrm{Com}_{b}$ and $\mathrm{Com}_{r}$. The formula for converting the $R G B$ color model to $\mathrm{LUMCom}_{b} \mathrm{Com}_{r}$ color model as in Eq. (19) (20) and (21). Here $L U M$ is luminance $\mathrm{Com}_{b}$ is component blue difference and $\mathrm{Com}_{r}$ is the component red red difference.

$$
\begin{gathered}
L U M=0.299 R+0.587 G+0.114 B(19) \\
\text { Com }_{b}=-0.169 R-0.331 G+0.500 B(20) \\
\text { Com }_{r}=0.500 R-0.419 G-0.081 B(21)
\end{gathered}
$$

Step2: For every pixel we will be having the $\operatorname{Com}_{b}$ and $\mathrm{Com}_{r}$ values. We will take $\mathrm{Com}_{b}$ and $\mathrm{Com}_{r}$ values, then calculate the mean of those values and also covariance matrix.

Step3: All types of skin colors have the same two-dimensional Gaussian model $G=(m, C)$, where $m$ speaks the mean and $C$ speaks the covariance matrix (as in Eq. (22) and (23)).

$$
m=E(X)=\frac{1}{n} \sum_{i=1}^{n} x_{i}
$$

$C=\mathrm{E}\left[(X-\mathrm{m})(X-m)^{T}\right]=\frac{1}{n} \sum_{i=1}^{n}\left(x_{i}-m\right)\left(x_{i}-m\right)^{T}$ (23) Where $x_{i}=\left(\operatorname{Com}_{b}, \operatorname{Com}_{r}\right)^{T}$ color values of pixel $i$ in the training samples, $n$ accounts the total number of color pixels. Example: after a statistic of 3051375 skin pixels from 200 color face images, we can get $\mathrm{m}$ and c values as in Eq. (24) and (25)

$$
\begin{gathered}
m=[117.4361,156.5599] \\
C=\left[\begin{array}{cc}
160.1301 & 12.1430 \\
12.1430 & 299.4574
\end{array}\right]
\end{gathered}
$$

Step4: In this skin color model, the probability of each pixel of query color image can be got, and the output of the image looks like that of skin. The probability is calculated as Eq. (26)

$$
p\left(\operatorname{Com}_{b}, \operatorname{Com}_{r}\right)=\exp \left[-0.5(x-m)^{T} C^{-1}(x-m)\right]
$$

Where $x=\left(\operatorname{Com}_{b}, \operatorname{Com}_{r}\right)$

Step5: if $p\left(\mathrm{Com}_{b}, \mathrm{Com}_{r}\right)$ is positive, indicates skin color pixel. Otherwise that pixel is not a skin pixel. Draw boxes around the skin pixels which are adjacent to one another. Don't draw the boxes for the skin pixels which are not adjacent. Find the area of all the boxes drawn. Extract the box with maximum area and project that portion. Hence the face is detected.

Step 6: So we can represent our model as the Skin Pixel Probability Model (SPPM). As we are finding whether a pixel is skin pixel are not based on the probability of that pixel.

End

\section{b. Template Based Approach for Face Recognition}

The Face Recognition system stages are clearly given below in the Figure 5. Even though there are many methods to recognize the face, we are using only Gabor based model because, The given image is viably multiplied with motivation reaction of the Gabor filters, the general equation is shown in equation (27), bringing about a progression of Gabor images with highlights, for example, edges and bars helpfully underlined for good segregation between facial appearances, So that, the faces with similar appearances will be retrieved to the user.

The detailed step by step process after the templates extraction is given is given in figure 6 . Before going to the process of template extraction, we need to understand what is meant by the Gabor filter. The detailed explanation for Gabor filter is given in the section 3.3.

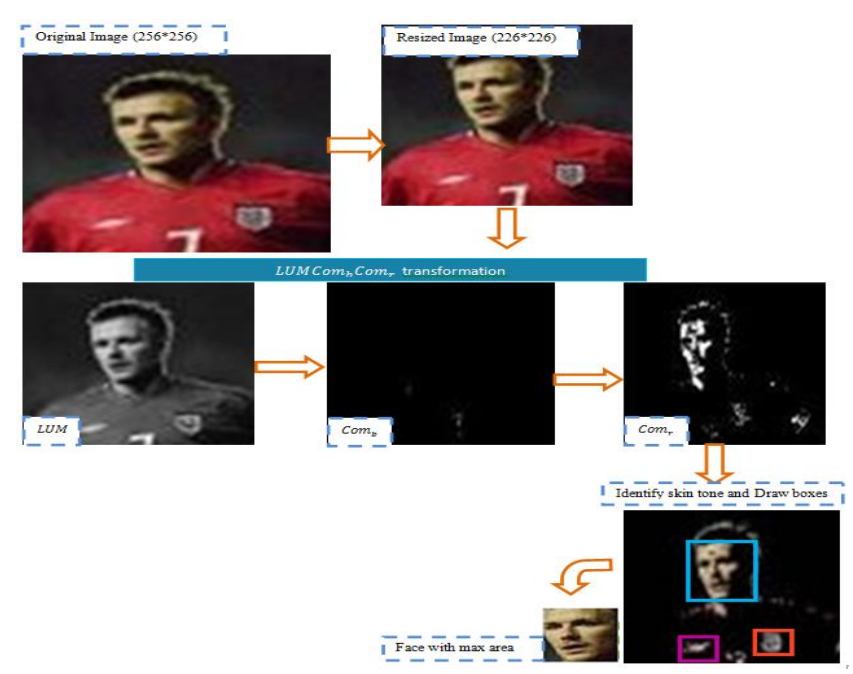

Figure 4 Proposed Face Detection System Stages

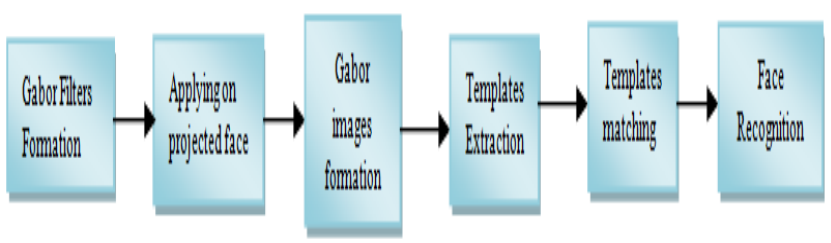

Figure 5 Proposed Face Recognition stages

\section{c. Gabor filters}

Gabor filters are a standout amongst the most ordinarily utilized surface descriptors for speaking to outward appearance data under changes on the face circumstances in past investigations. In this paper, 2D Gabor filter [20] is embraced and it very well may be numerically communicated as in eq 27 and 28.

$$
\begin{gathered}
F(x, y)=\exp \left(-\frac{X^{2}+\gamma^{2} Y^{2}}{2 \sigma^{2}}\right) \times \cos \left(\frac{2 \pi}{\lambda} X\right) \\
X=x \cos \theta+y \sin \theta, Y=-x \sin \theta+y \cos \theta \\
\text { where, orientation } \theta \text {, the viable width } \sigma \text {, the }
\end{gathered}
$$
wavelength $\lambda$, the perspective proportion $\gamma$. Rather than the broadly utilized five scales, eight scales (are embraced here to test the outcomes utilizing a bigger number of scales. We used 8 different $\sigma$ values ranging from 5 to 40 $(5,10,15,20,25,30,35,40)$. Subsequently, four orientations $\left(-45^{\circ}, 90^{\circ}, 45^{\circ}, 0^{\circ}\right)$ are utilized. And the other remaining parameters are kept as constant. For the values of,$\lambda$, we used $0.25,30$ respectively as constant. The given image is viably convolved with motivation reaction of the Gabor filters as in equation (28), bringing about a progression of Gabor images with highlights, for example, bars and edges helpfully underlined for better segregating between facial appearances.

\section{d. Extraction of Template}

Template extraction receives the given algorithm to gather a lot of templates (As appeared in Figure 8). In this paper, four templates sizes are utilized: $2 \times 2,4 \times 4,6 \times 6,8 \times 8$.

Published By: 
These templates are the two dimensional windows which will be super imposed on the Gabor images of particular scale. A detected face can have 32 Gabor images. These Gabor images will be dived into groups of four in each group. Each group is called as a scale. Each scale will consist of 4 Gabor images. The templates will be superimposed on the Gabor image at a particular location(x,y) of the Gabor image. One template will extract the pixel values from the 4 different orientations of the same scale. The pixel values are extracted for each template will be stored based on the number of scale. The templates will be applied on all the Gabor images obtained which belongs to all 8 scales and 4 orientations. The extracted values of each template from 4 orientations will be collected and then the average for each template is calculated. So for one scale we will be getting the $4 * 4$ i.e 16 templates. So for all these 16 templates we will be getting the 16 averages. When these 16 averages are combined together, we will get one average known as Scale Average. As we know there are 8 scales, then we can have 8 Scale Averages We keep these average values in one vector. So for every image there will be a vector containing the 8 scale average values in it and for every scale we can have a vector storing average values of $4 P_{j} \times P_{j}$ templates. And for every $P_{j} \times P_{j}$ template we can have a vector storing the average values of $O_{\text {num }}$. We use Euclidean distance for similarity matching. In order to understand about how the template is being extracted, we need to understand what is happening at various levels. The template extraction involves various levels. The first level is the Scale level and second level is the Orientation level and the third level is the Template level. Now the paragraph below will explain what is happening at various levels.
Scale level: After the convolution of Gabor filters with the Query image, we will get the Gabor images. These Gabor images are divided into scales. There are totally eight scales. These eight scales we got because we used 8 different values for $\sigma$. These eight scales will consist 32 the Gabor images. We divided the total 32 Gabor images into 8 parts. So that each part will contain the 4 Gabor images.

Orientation level: At this level we have four Gabor images for each scale. These four Gabor images of each scale are formed with the four orientation values that we have used.

Template level: At this level we will apply 4 templates of different sizes on the Gabor images of different orientations. At this level, the templates will get convolved with the Gabor images. The various levels are shown in the figure 6 below. From the top to bottom we can observe the various levels in the figure 6. Scale level is the highest level and template level is the lowest level. We combine all the averages obtained in various levels (as shown in equation 29). The average obtained here represents the image average. We compare the similarity of images based on this average only.

$$
s(x, y)=\frac{1}{8} \sum_{i=1}^{8} \frac{1}{4} \sum_{j=1}^{4} \frac{1}{4} \sum_{k=1}^{4} x_{i}
$$

Figure 7 shows how the query image goes under various stages. As we can observe in the image, firstly the Gabor images are formed for query image. Then Templates will be applied on those Gabor images. These template are just the two dimensional windows which will extract the content of the image.

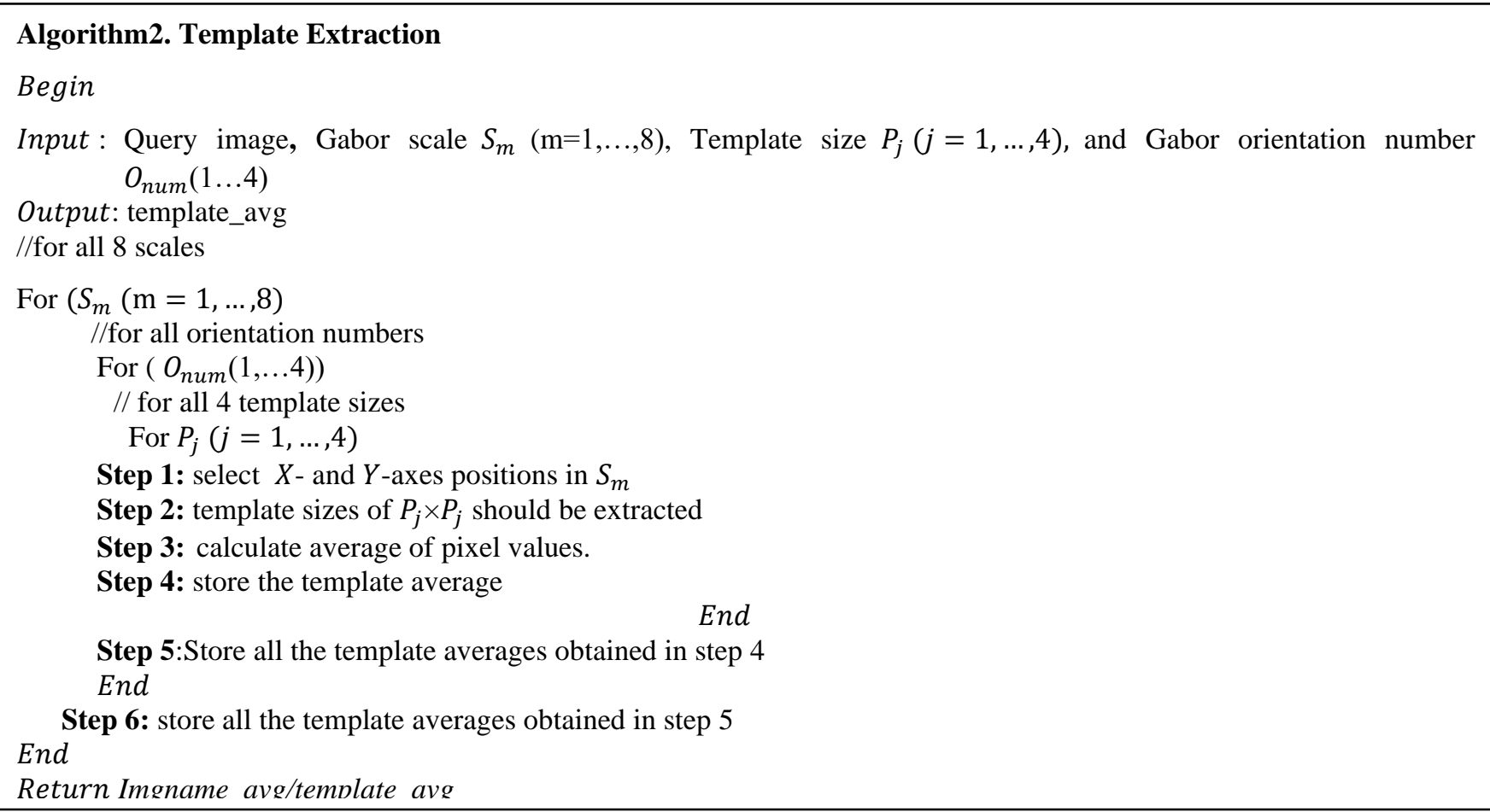




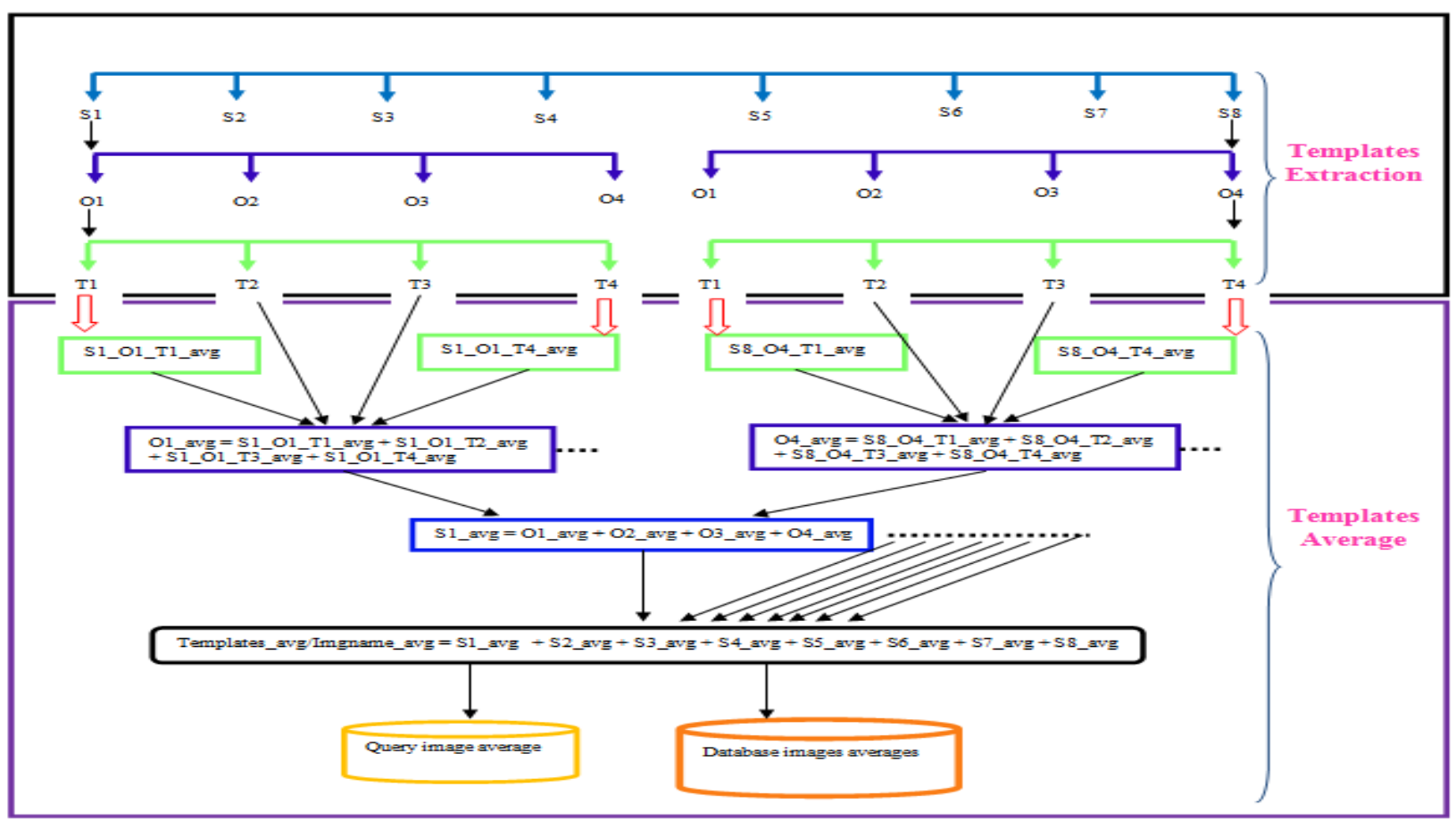

Figure 6 Flow of finding an image average

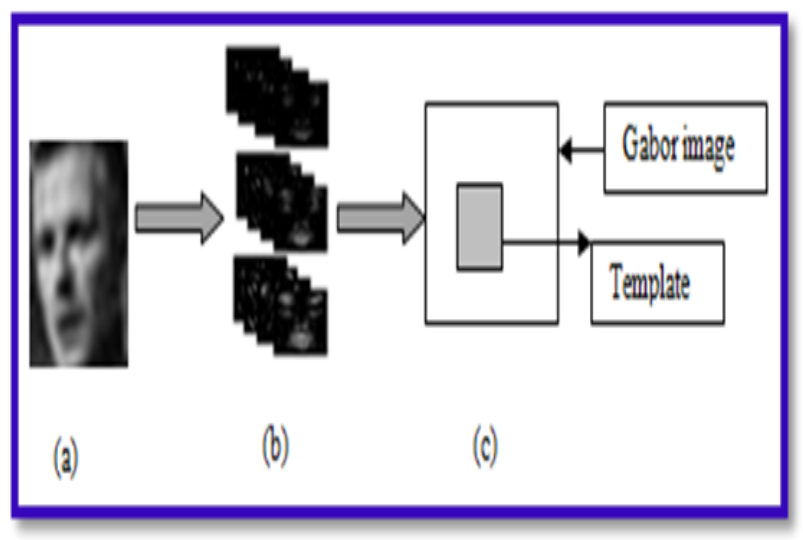

Figure 7 from left to right: (a) Query image, (b) Gabor images of 3 scales, each scale with four orientations (c) only one scale is taken, showing a template being selected from $i^{\text {th }}$ orientation at $(X, Y)$ location.

\section{B. Similarity Measure Stage}

In this stage, we compared the average value obtained for query image with the average values present in the database. For this purpose we have used Euclidean distance. Now in 3.5 section we see how we compare the average values.

\section{a. Template matching}

Template matching is used to match the input image content with the dataset images content. We get the average values for all the templates and for all the 8 scales. The final average value that we got by computing the average values of all scales is compared with the database images average values. The equation for finding the similarity in terms of average is shows in Equation (30). Based on the Euclidean formula, for similarity matching we used the equation (30). As we know that we get 4 templates for each Gabor image, and also each scale contains 4 Gabor images with each Gabor image containing the 4 templates, and we find the average for each template.

$$
d(I, Q)=\sqrt{\sum_{i=1}^{n}\left(I_{i}-Q_{i}\right)^{2}}
$$

Based on the Threshold value that we prefer, matched images will be shown to the user. In those images the user has to select the images which are mostly related to the query image.

\section{Display Result Image Set Stage}

After the completion of similarity measure stage, the third stage that we come across is, Display Result Image Set Stage. In this stage the similar images will be displayed to the user. These images will be displayed on a window called Display Window. 10 images will be displayed to the user for each iteration, because we have set the limit of Display window to 10 only. This is because 10 images can be clearly viewable on the Display Window and More images on the display window seem to be clumsy and user feels unpleasant.

\section{Feedback Process Stage}

After the similar images are displayed then the user will be asked for the feedback. The system will collect the most common images based the feedback response given by the user. The user is given a chance to give the feedback based on the most similar images will be given most priority and the less similar images will be given less priority. The user will freely give the number to the images which he is giving as a feedback to the system. The system will recognize the most similar image to the query image if that's image number value is less. Note that numbers will start from 1 . The most similar image will be given 1 number and then the next most similar image will be given as 2, this process of giving the feedback continues according to the user interest. If the user finds the 10 most similar images, then he can give the number from 1 to 10 . We should keep in mind that there will be a chance that, a single user will have different images with different expressions.

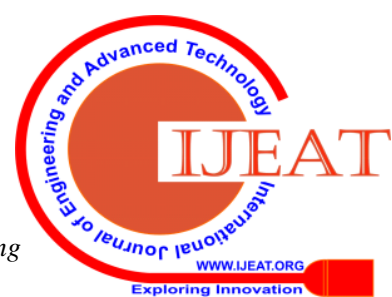


So the user has to keep in mind that he is working on a system where he can get the images of one person. Whenever the system displays the images to the user, which are not related to the query image given, then the user should mention them as irrelevant, by giving -1 number to them, and the relevant images will be given feedback based on their most similarity with the query image. We should note that the images assigned with -1 are not related to the query image. The images assigned positive values with them are treated as relevant images.

\section{EXPERIMENT SETUP}

In our experiment, we have used the PubFig Database. The PubFig database is a large, real-world face dataset consisting of 58,797 images of 200 people collected from the internet. Unlike most other existing face datasets, these images are taken in completely uncontrolled situations with non-cooperative subjects. Thus, there is large variation in pose, lighting, expression, scene, camera, imaging conditions and parameters, etc. Figure 8 shows some of the images in Pub Fig database with variation in pose, lightning, expression, and camera variations.

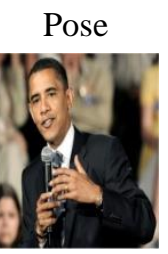
Lightning

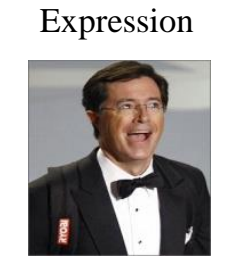

Camera
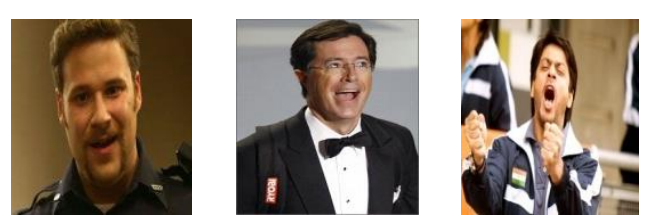

Figure8. Images in PubFig database

Our database contains 400 images. It is divided into two parts. The first part contains development set images and the second part contains the evaluation set images. The development set and evaluation set contains 200 images. In development set and evaluation set we have 10 people. For 10 people we have taken 20 images with some variations in the appearance of the image.

The experimentation was performed on the Windows platform powered by a Core 2 duo processor $2.6 \mathrm{GHz}$ CPU using $4 \mathrm{~GB}$ of RAM. The prototype system is implemented using Opencv.

\section{PERFORMANCE ANALYSIS}

Here in Table 1 we can see the experimental results for our Face detection method. Our method gave us good results in detecting the face. Here in the Table 1 it can be seen that, the identification value for one-face image is 94 percent. In Table 2 we can see the comparison of algorithms. When we compare our method with the Hewa Majeed [21] we can find that our method is better than that. This similarity shows that the demonstrated calculation gives best outcomes.

Table 1 Total outcomes of the experiment

\begin{tabular}{|c|c|c|c|c|}
\hline $\begin{array}{c}\text { Type of } \\
\text { Image }\end{array}$ & $\begin{array}{c}\text { No of } \\
\text { faces }\end{array}$ & $\begin{array}{c}\text { Exactly } \\
\text { detected } \\
\text { faces }\end{array}$ & $\begin{array}{c}\text { lost } \\
\text { faces }\end{array}$ & $\begin{array}{c}\text { Rate of } \\
\text { Detection } \\
\text { (\%) }\end{array}$ \\
\hline $\begin{array}{c}\text { One } \\
\text { face }\end{array}$ & 100 & 94 & 6 & 94.0 \\
\hline
\end{tabular}

Table 2 Comparison between different methods

\begin{tabular}{|c|c|c|c|c|c|}
\hline $\begin{array}{c}\text { Criteri } \\
\text { on }\end{array}$ & $\begin{array}{c}\text { RG } \\
\text { B } \\
\text { Spa } \\
\text { ce } \\
{[22]}\end{array}$ & $\begin{array}{c}\text { YCb } \\
\text { Cr } \\
\text { Color } \\
{[22]}\end{array}$ & $\begin{array}{c}\text { HSI } \\
\text { Spa } \\
\text { ce } \\
{[22]}\end{array}$ & $\begin{array}{c}\text { Hewa } \\
\text { Majeed[ } \\
\text { 21] }\end{array}$ & $\begin{array}{c}\text { Demonstra } \\
\text { ted method }\end{array}$ \\
\hline $\begin{array}{c}\text { Accura } \\
\text { cy }\end{array}$ & $\begin{array}{c}21.6 \\
4 \%\end{array}$ & $\begin{array}{c}84.61 \\
\%\end{array}$ & $\begin{array}{c}73.8 \\
\%\end{array}$ & $92.69 \%$ & $94 \%$ \\
\hline
\end{tabular}

Table 3 and 4 shows the performance analysis for face recognition. We evaluated the System's performance with the Average precision. The formula for average precision is given below in equation no (30).

$$
\text { Precision }=\frac{A}{A+B} \times 100
$$

Where A gives the number of relevant images accessed and $B$ gives the number of irrelevant images accessed

The system's working is evaluated for each input image and their corresponding precision value is calculated using the above equation. From the Table 3 shown below, we can clearly observe that our approach is well enough to retrieve more similar face images and that it also decreased the retrieval of false positive images when compared with its counterpart skin color based and template based approaches. Figure 9 gives the Precision graph of proposed method with other methods.

Table 3: Precision values of proposed method with other methods.

\begin{tabular}{|c|c|c|c|}
\hline Iterations & $\begin{array}{c}\text { Skin } \\
\text { Color } \\
\text { based }\end{array}$ & $\begin{array}{c}\text { Template } \\
\text { based }\end{array}$ & $\begin{array}{c}\text { Proposed } \\
\text { Approach(FRSTR) }\end{array}$ \\
\hline 1 & 0.088 & 0.138 & 0.126425 \\
\hline 2 & 0.09428 & 0.14428 & 0.19625 \\
\hline 3 & 0.106424 & 0.156424 & 0.229426 \\
\hline 4 & 0.111579 & 0.161579 & 0.250526 \\
\hline 5 & 0.122546 & 0.172546 & 0.268985 \\
\hline 6 & 0.135 & 0.185 & 0.293378 \\
\hline 7 & 0.155 & 0.205 & 0.33704 \\
\hline 8 & 0.178899 & 0.228899 & 0.382095 \\
\hline 9 & 0.22883 & 0.278899 & 0.433161 \\
\hline 10 & 0.26226 & 0.31226 & 0.480132 \\
\hline
\end{tabular}

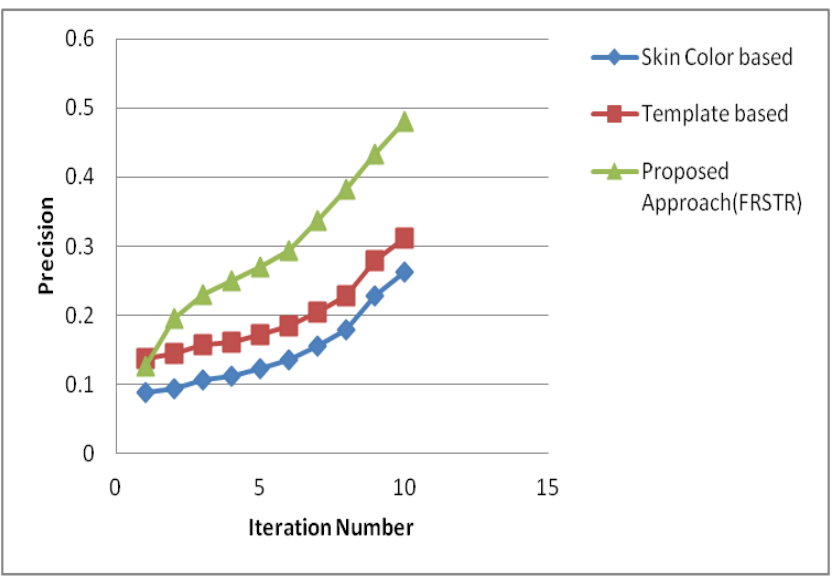

Figure 9: Precision graph of proposed method with other methods. 


\section{CONCLUSION}

This article demonstrates a face recognition method using skin color and templates. This method works effectively even though there are some illumination issues also. We used two differing color spaces RGB, and YCbCr for face detection. We have used CLAHE method [24] for illumination compensation. We utilized the images present in the PubFig database. For our experiment we took the images having single faces from the PubFig database, and made a dataset of faces. We used the two databases based on the requirement. The first database will contain the images of PubFig images while the second database contains the extracted faces from the images in first database. We used first database for detecting the face and the second database for recognizing the face. Finally it was found that, our system gives good results when compared with other methods. Time that was taken on PubFig pictures tells that the demonstrated figuring is speedy and 4 test pictures can be taken care of consistently on a Intel core2duo $3.2 \mathrm{GHz}$ machine.

\section{REFERENCES}

1. Pantic M, Zeng Z, Huang TS (2009), Roisman GI, : A Survey of Affect Recognition Methods:Audio, Visual, and Spontaneous Expressions. Pattern Analysis and Machine Intelligence, IEEE Tran.on 31 (1):39-58

2. Slater M (2007), Towner H,: Reconstruction and Recognition of Occluded Facial Expressions Using PCA. In: Affective Computing and Intelligent Interaction. pp 36-47

3. Chibelushi, C.C., Bourel F, Low, A.A. Recognition of facial expressions in the presence of occlusion. In: 12th British Machine Vision Conf, 2001. pp 213-222

4. Chibelushi CC, Bourel F, :Low AA Robust facial expression recognition using a state-based model of spatially-localised facial dynamics. In: Automatic Face and Gesture Recog., 2002.Proc. Fifth IEEE International Conf on, 2002. pp 106-111

5. Ranganath S, Tan Dat N, : Tracking facial features under occlusions and recognizing facial expressions in sign language. In: Automatic Face \& Gesture Recogn, 2008. FG '08. 8th IEEEInternational Conf on, 2008. pp 1-7

6. Arguin M, Hammal Z, Gosselin F (2009): Comparing a novel model based on the transferable belief model with humans during the recognition of partially occluded facial expressions. In Journal of Vision 9 (2):1-19

7. Kang H, YuLi X, Xia M, Zheng L, ShanWei L Robust facial expression recognition based on RPCA and AdaBoost. In: Image Analysis for Multimedia Interactive Services, 2009. WIAMIS '09.

8. Jia K-b (2011), Jiang B, :Research of Robust Facial Expression Recognition under Facial Occlusion Condition. In: Active Media Technology, vol 6890. Lecture Notes in Computer Science. Springer Berlin/Heidelberg, pp 92-100

9. Kotsia I, Pitas I, Buciu I, : Facial expression analysis under partial occlusion. In: Acoustics, peech, and Signal Processing, 2005. Proc. (ICASSP '05). IEEE International Conf on, 2005. pp 453-456

10. Buciu I, Kotsia I, Pitas I (2008) An analysis of facial expression recognition under partial facial image occlusion. Image and Vision Computing 26 (7):1052-1067

11. Bassili JN (1979) Emotion recognition: The role of facial movement and the relative importance of upper and lower areas of the face. Journal of Personality and Social Psychology 37

12. Bülthoff HH (2008), Cunningham DW, Nusseck M, Wallraven C, :The contribution of different facial regions to the recognition of conversational expressions. Journal of Vision 8(8):1, 1-23

13. Singh, D. S. Chauhan, Mayank Vatsa and Richa Singh, Sanjay Kr, "A Robust Skin Color Based Face detection Algorithm," Tamkang Journal of Science and Engineering, vol. 6 (4), pp. 227-234, 2003.

14. MuhammadTariq:"Face Detection by Image Discriminating," 2006. [Online].

Available:Http://www.bth.se/fou/cuppsats.nsf/all/6c509ae86a297ca4 c12571d300512cac/\$file/DVD009-MasterThesisReport.pdf.

15. Zihong Fan and Michael Padilla: "Automatic Face Detection Using Color Based Segmentation and Template / Energy Thresholding,"
2003.

[Online].

Available:

http://www.stanford.edu/class/ee368/Project_03/Project/reports/ee36 8group16.pdf.

16. B. Yegnanarayana, 1999. :Artificial Neural Networks, Prentice-Hall of India, New Delhi.

17. T. Kohonen, 1988: Self-Organization and Associative Memory, Springer-Verlag, Newyork.

18. T. J. Stonham, 1984. :Practical Face Recognition and Verification with WISARD, In Aspects of Face Processing, pp.426-441.

19. G. W. Cottrell , D. Demers 1993. :Non-linear Dimensionality Reduction, In Advances in Neural Information Processing Systems, pp.580-587.

20. Gabor D (1946) Theory of communication. Institution of Electrical Engineers -- Journal --Radio and Comm Engineering 93 (26, Part III):429-457.

21. Hewa Majeed Zangana1, Imad Fakhri Al-Shaikhli2: A New Algorithm for Human Face Detection Using Skin Color Tone: IOSR Journal of Computer Engineering (IOSR-JCE) e-ISSN: 2278-0661, p-ISSN: 2278-8727Volume 11, Issue 6 (May. - Jun. 2013), PP 31-38.

22. Raghuvanshi, D. S. and Agrawal, D., "Human Face Detection by using Skin Color Segmentation, Face Features and Regions Properties," International Journal of Computer Applications, vol. 38No.9, 2012.

23. SubrahmanyamMurala, R. P. Maheshwari, and R. Balasubramanian, "Local Tetra Patterns: A New Feature Descriptor for Content-Based Image Retrieval", IEEE Transactions on Image Processing, vol. 21, no. 5, 2874-2886, May 2012.

24. https://stackoverflow.com/questions/24341114/simple-illuminationcorrection-in-images-opencv-c.

\section{AUTHORS PROFILE}

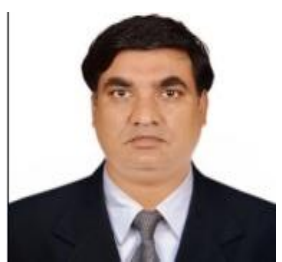

Dr. T. Venugopal, is currently working as a Professor \& Head Department of CSE at JNTUH College of Engineering, Nachupally, Jagtial District, Telangana, India. He also rendered his services as Training and Placement Officer and NSS program officer at this institute. He has rich experience as a teacher and transformed many students by his technical and motivational skills.. His interesting areas include image processing, data mining, cryptography. Under his guidance 10 students have obtained Ph.D. and about 17 students are pursuing Ph.D. He has authored a text book on Design and Analysis of Algorithms. He has published more than 60 research papers in various International Journals. He participated and presented research papers in International and National Conferences. He has organized and participated in workshops/ seminars and training programs. He is honoured with "The Vishista Seva Puraskar" by J.N.T.U.H College of engineering, Nachupalli, Jagitial for his meritorious services rendered to this Institution during 2009-10. He has obtained his BE in ECE from the Osmania University, Hyderabad, Telangana, India. M.Tech in CSE and PhD in CSE from the JNTUH University, Hyderabad, Telangana, India.

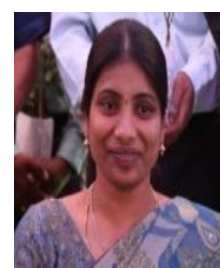

T. Archana, is working as Assistant Professor in CSE, UCE, Kakatiya University, Kothagudem, District. Bhadradri Kothagudem, Telangana, India. She has obtained her B.Tech (CSE) from KITS, Huzurabad, Dist. Karimnagar, Telangana, M.Tech in CSE from JNTU Ananthapur, Dist. Ananthapur, A.P., India, She is pursuing her Ph.D from JNTUH University, Hyderabad, Telangana, India. She has about 15 years of teaching experience. Her interested area are image processing, networks. 